\title{
Temperature and Pinning Effects on Driving a 2D Electron System on a Helium Film: A Numerical Study
}

\author{
Pablo F. Damasceno • Cláudio José DaSilva • \\ José Pedro Rino • Ladir Cândido
}

Received: 20 January 2010 / Accepted: 15 April 2010 / Published online: 29 April 2010

(C) Springer Science+Business Media, LLC 2010

\begin{abstract}
Using numerical simulations we investigated the dynamic response to an externally driven force of a classical two-dimensional (2D) electron system on a helium film at finite temperatures. A potential barrier located at the center of the system behaves as a pinning center that results in an insulator state below a threshold driving force. We have found that the current-voltage characteristic obeys the scaling relation $I=f^{\xi}$, with $\xi$ ranging from $\sim(1.0-1.7)$ for different pinning strengths and temperatures. Our results may be used to understand the spread range of $\xi$ in experiments with typical characteristic of plastic depinning.
\end{abstract}

Keywords Collective transport · Electrons in helium · Non-linear phenomena

\section{Introduction}

The phenomena of collective transport in strongly correlated two-dimensional (2D) electron systems has been a subject of increasing theoretical and experimental interest since the discovery of the fractional quantum Hall effect (FQHE) [1] and of

Present address:

P.F. Damasceno $(\bowtie)$

Applied Physics Department, The University of Michigan, Ann Arbor, MI 48109-1040, USA

e-mail: damascus@umich.edu

P.F. Damasceno - C.J. DaSilva · J.P. Rino

Departamento de Física, Universidade Federal de São Carlos, 13.565-905, São Carlos, São Paulo, Brazil

L. Cândido

Instituto de Física, Universidade Federal de Goiás, C.P.131, 74.001-970, Goiânia GO, Brazil

L. Cândido

Instituto de Física de São Carlos, Universidade de São Paulo, 13560-970, São Carlos, São Paulo, Brazil 
metal-insulator transitions (MIT) in 2D [2, 3], first reported for a Si metal-oxidesemiconductor field-effect transistor (MOSFET) by Kravchenko et al. [4]. Until now, the latter is not well understood and is currently focus of intense research. It was found that the MIT shows nonlinear behavior in current versus voltage $(I-V)$ curves and a great deal of effort has been spent to understand it [5-7]. Such ordered systems, composed of strongly interacting electrons driven by an external force, can exhibit a variety of interesting critical phenomena [8]. Striking examples are pinning and depinning on vortex matter [9] and charge-density-waves (CDW) conductors [10], charge transport in metallic quantum dots [11], Coulomb-blockade in conducting nanomaterials [12-14], nonlinear magnetoconductivity of 2D Wigner crystals in ${ }^{4} \mathrm{He}$ superfluid $[15,16]$.

In all these systems, one can scale the $I-V$ characteristic curves using the form $I=f^{\xi}$, where $f=\left(V-V_{t}\right) / V_{t}$ is a reduced voltage [17]. The exponent $\xi$ has a wide range of values both theoretically and experimentally. The value of the exponent $\xi$ depends on the kind of flow produced in these systems when they are dragged by the external force. Analytically, one expects $\xi<1$ when the system starts to flow elastically, which was confirmed by numerical experiments [18]. In the case of a plastic flow, numerical calculations seem to agree with $\xi>1$ in 2D [18, 19]. In general, analytic results of charge transport studies predict $\xi=1.0$ for one-dimensional (1D) arrays and $\xi=5 / 3$ for the $2 \mathrm{D}$ case. For numerical calculations, $\xi=2.0$ [11] while experimentally it ranges from $\xi=1.3$ to 2.5 [20, 21]. In the last studies, electron transport was analyzed on a two-dimensional array of metallic dots fabricated with different degrees of disorder. In summary, theory and experiment have no consensus on how the strength of disorder affects the nonlinear $(I-V)$ characteristics. Furthermore, it is not yet clearly understood which effects are responsible for the pinning dynamics in these systems [13].

One interesting question to consider is what kind of theoretical models are best suited to study the depinning mechanism that could clarify the behavior of $\xi$. There are a variety of models of driven disordered systems for both regimes, elastic and plastic [22-24]. In addition, some models were proposed to numerically study the charge transport in 2D arrays in the presence of quenched disorder [25, 26]. This is motivated by the fact that the charge flow at depinning resembles what has been seen in other systems such as flux lattices. In those models, the quenched disorder may be created by introducing pinning centers with, for instance, Gaussian or parabolic shapes. These pinning centers mimic barriers that the electrons must overcome in order to produce any collective charge transport. Analogously, in the experiments cited above a charge must overcome a barrier to travel between the dots. Furthermore, the charging of the underlying background is another way to create a system with quenched disorder [27].

In this work, we examine a periodic electronic structure in the presence of strong barriers that lead to plastic depinning, i.e., the crystal lattice periodicity is broken and consequently the translation symmetry is lost $[8,28]$. The comparison between simulated, theoretical and experimental critical exponents is discussed for such regime at different temperatures and pinning strengths. In order to test this, we have used Langevin Molecular Dynamics simulations to study the dynamical response to an external driving force of classical 2D electrons in helium film. This system has received 
much attention for almost three decades. Theory and experiment [29, 30], together with numerical calculations [31, 42], showed exciting importance for the understanding low dimensionality picture, which has been renewed by recent experimental results on the dynamic flow of surface electrons over liquid helium [32-34].

\section{Model and Computational Details}

The model we have studied here is a prototype of an ideal $2 \mathrm{D}$ electron system. $N$ classical electrons with charge $e$ are adsorbed on a film of liquid helium with dielectric constant $\epsilon$ coating a solid substrate with dielectric constant $\epsilon_{s}$. In the present case, the particles have coordinates $r=(x, y)$ and interact through a screened Coulomb potential [31]

$$
V(r)=e^{2}\left(\frac{1}{r}-\frac{\delta}{\sqrt{r^{2}+4 d^{2}}}\right),
$$

which is controlled by the film thickness $d$ and the parameter

$$
\delta=\frac{\epsilon_{s}-\epsilon}{\epsilon_{s}+\epsilon} .
$$

In order to examine the collective transport characteristics, as well as the nonlinear dynamics in this system, we have constrained the $2 \mathrm{D}$ electrons by a potential barrier

$$
V_{c}(x, y)=\frac{V_{0}}{1+\alpha^{2} x^{2}+\beta^{2} y^{2}}
$$

centered at the origin. The parameters $\alpha$ and $\beta$ determine the shape of the Lorentzian potential and its strength is given by $V_{0}$. This barrier can be experimentally realized by introducing either one central or a quasi one-dimensional array of microelectrodes submerged into the helium and attached to the substrate. This creates an electronic trap with a repulsive (attractive) gate (reservoir) potential [35].

To simulate the system, we integrated the Langevin equation of motion

$$
m \ddot{r}=-\nabla U(r)-b m \dot{r}+\zeta(t),
$$

where

$$
U=V+V_{c}
$$

$b$ is the friction coefficient, and $\zeta(t)$ is a random force vector that mimics thermal noise and has a zero mean

$$
\left\langle\zeta_{i}(t)\right\rangle=0
$$

and standard deviation

$$
\left\langle\zeta_{i}(t) \zeta_{j}\left(t^{\prime}\right)\right\rangle=2 m b k_{B} T \delta_{i j} \delta\left(t-t^{\prime}\right)
$$


A constant external driving force $F$ is applied parallel to the $x$-direction to simulate the effect of an external electric field.

The simulations were performed in a rectangular box with area $A$ for a fixed density of $2.2 \times 10^{9} \mathrm{e} / \mathrm{cm}^{2}$. The sides of the simulation cell have a ratio of $\sqrt{3} / 2$ to allow for $4 M^{2}$ electrons to lie in a perfect triangular lattice at zero temperature and zero applied field. In addition, we used periodic boundary conditions and the electron-electron interaction is handled with the Ewald summation. Throughout this paper, we use $\sqrt{A}$ and $E_{0}=e^{2} / 2 \sqrt{A}$ as units of length and energy, corresponding to 4.26 micrometer and $2.70 \times 10^{-16} \mathrm{erg}$, respectively. These reduced quantities will be denoted by an " $*$ ". The effect of temperature on the dynamical response of the electrons to an external force in this system is determined by carefully selecting systems so as to pick up the essential physics with temperatures varying from 1 to $100 \mathrm{mK}$, $d=100 \AA, \delta=0.5789$ (glass substrate), $\alpha^{*}=2, \beta^{*}=30$ and $N=400$ electrons. The driving force was increased starting from zero and at each value of $F^{*}$ we measure the electron velocity in the $x$-direction

$$
v_{x}^{*}=\frac{1}{N} \sum_{i} \mathbf{v}_{i}^{*} \cdot \hat{x}
$$

after $10^{6}$ simulation steps. The average electron velocity $v_{x}^{*}$ and the external force $F^{*}$ may be directly related to the current $(I)$ and voltage $(V)$ in dc experiments, respectively. We shall further refer to $v_{x}^{*}-F^{*}$ as current-voltage curves.

\section{Numerical Results and Discussion}

An important aspect about universality in critical phenomena is that systems within an universality class have the same critical exponents and scaling functions, therefore the critical exponent is independent of the system details [22]. In previous works we have studied the behavior of the critical exponent for the same system described above as a function of the type of substrate and helium film thickness [36] as well as of the shape of the barrier [43]. In neither cases considerable changes on the value of the exponent were found, that remained constant and approximately 1.3.

In what follows, we have studied the critical exponent's behavior as a function of temperature and disorder strength for the system described in the previous section. It is important to mention that the values of critical exponents obtained from our simulation are comparable with those from experiments and theory in the plastic depinning regime. Although during the presentation of the results we will make comparisons between these so different systems, it is out of scope of this paper, however, try to explain the physics similarities between them.

In Fig. 1 we show the driving threshold $F_{c}^{*}$ as a function of potential barrier height $V_{0}^{*}$ at two fixed values of temperatures, $T=0$ (circles) and $T=10 \mathrm{mK}$ (squares). The thermal rounding on the threshold is treated using the same technique used in Ref. [12], which allows us to estimate the critical force $F_{c}^{*}$. The overall increase in the threshold is consistent with the increasing average force for the electrons to overcome the barrier. It is clear that the threshold shifts to lower values when $T$ increases, 
Fig. 1 (Color online) Threshold value of the driving force as a function of the barrier height $V_{0}^{*}$ for $T=0$ (circles) and $T=10 \mathrm{mK}$ (squares). The dotted lines are a power law fit. Inset: Drift velocity as function of driving force for $V_{0}^{*}=60$

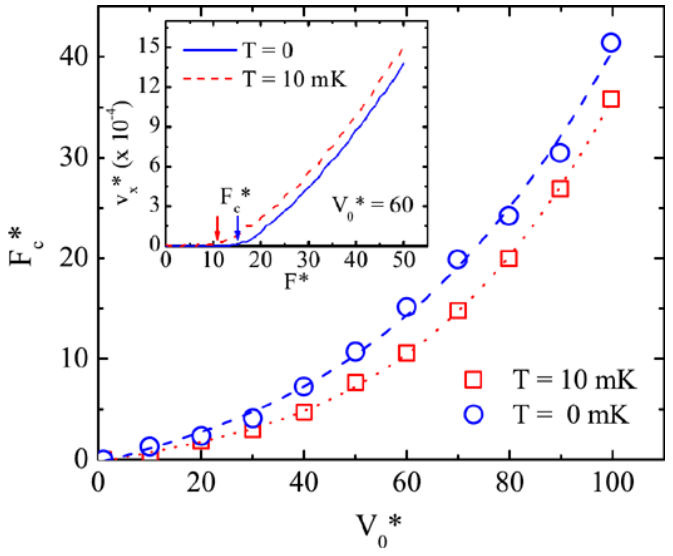

indicating that finite temperature reduces the effect of the barrier (directly associated with the strength of disorder). The thermal energy acquired by the electrons favors their motion through the barrier and the critical driving force decreases. The inset in Fig. 1 shows $v_{x}^{*}$ vs $F^{*}$ (current-voltage curves) for the same two values of temperature and $V_{0}^{*}=60$ (the arrows indicate the location of the threshold). Here, we note that the curves do not change their shape when the temperature increases. This behavior indicates that the scaling exponent should be independent of temperature for this specific value of disorder strength.

The temperature dependence of the driving threshold $F_{c}^{*}(T)$, for a fixed value of $V_{0}^{*}=60$, is shown in Fig. 2. We have found that for $T \geq 10 \mathrm{mK}$ the threshold decreases, with increasing temperature, in the same way as observed experimentally in nonlinear systems obeying a power-law $F_{c}^{*} \sim T^{-\gamma}$ with $\gamma=0.8$. The critical exponent, however, seemed to be dependent on the details of the system and the disorder involved. With our simulations we have verified that, for $T \gtrsim 40 \mathrm{mK}$ the threshold is almost zero and this clearly indicates that there is no tendency for the threshold to saturate as a function of temperature. Our $F_{c}^{*}(T)$ results indicate that electrons on a helium film give a scaling exponent close to that measured in quasione-dimensional polymer nanofibers $(\gamma=1.1)[12,13]$, where the authors did not observe any tendency of saturation. Figure 2(b) shows the current $\left(v_{x}^{*}\right)$ as a function of temperature for different voltage values $\left(F^{*}\right)$. As one can see, at high temperatures and low voltages there is a strong temperature dependence of the current, while at low temperatures and high voltages the current-voltage characteristic is almost temperature independent. In general, the observed behavior is particularly similar to those obtained in some Coulomb-blockade transport [12] and non-Ohmic conduction [37] experiments. One can also see that the curves in Fig. 2(b) exhibit a behavior resembling that observed in 2D MIT numerical [38] and experimental [39] results.

In Fig. 3 we elaborate further on the current as a function of temperature. For systems at low voltages, i.e., $F^{*} \leq 3$, our results fitt an Arrhenius form

$$
I \propto \exp \left(-E_{a}^{*} / k_{B} T\right)
$$

where $E_{a}^{*}$ is the energy necessary to activate the current, see Fig. 3(a). We found that the activation energy to be of the order of $\sim 51-64$ in the voltage range $2-3$, 
Fig. 2 (Color online)

(a) Temperature dependence of the threshold value of the driving force for a barrier with $V_{0}^{*}=60$. (b) Temperature dependence of current $v_{x}^{*}$ at different voltages $F^{*}$ for the same value of potential barrier

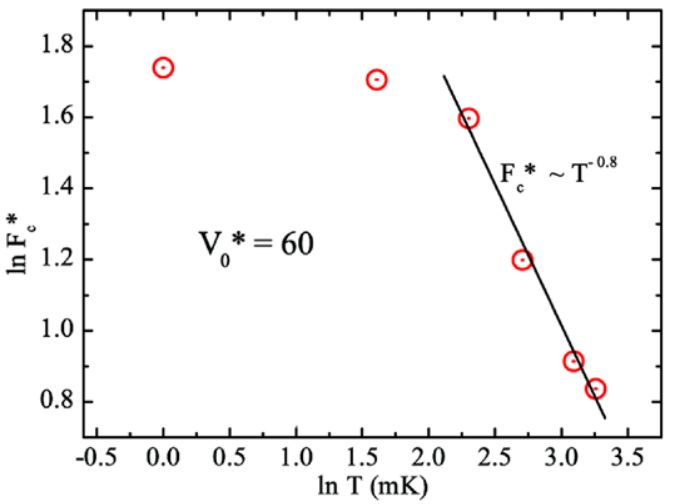

(a)

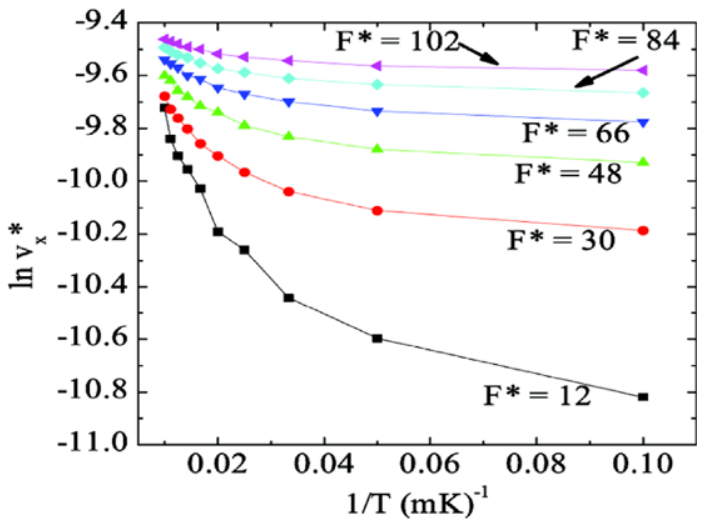

(b)

which is close to the pinning energy $V_{0}^{\star}=60$. This agreement between the pinning and activation energies shows that electron transport is determined by thermal activation mechanism in this range of temperature and voltage. By increasing the voltage further, i.e., for $F^{\star} \geq 6$, the activation energy decreases and approaches to $k_{B} T$. In summary, for low voltages the thermal motion represents a key factor in the process of overcoming the barrier and therefore such regime is completely described by Arrhenius law (Fig. 3(b)). For high voltages (Fig. 3(c)), however, the process becomes temperature independent and the deviation is expected once the overcoming process is entirely resulting from the driven force. Finally, (Fig. 3(d)) shows that the channels structure can be completely destroyed even for high voltages if the temperature is large enough.

Figures 4 and 5 demonstrate the effect of temperature and disorder (barrier height) on the scaling behavior of depinning. The scaling exponent $\xi$ is given by $\xi=\ln v_{x} / \ln f^{*}$, where $f^{*}=\left(F^{*}-F_{c}^{*}\right) / F_{c}^{*}$ is a reduced variable. We have found the critical exponent value as $\xi \sim 1.36 \pm 0.03$ in the interval of temperature varying from $T=0$ to $25 \mathrm{mK}$ at fixed barrier height $V_{0}^{*}=60$, as shown in the inset of Fig. 4 . For relatively weak disorder, the scaling exponent is weakly dependent on temperature. The obtained values of $\xi$ in this range of temperatures for this specific barrier 
Fig. 3 (Color online) (a) The temperature dependence of the current for some values of the voltage with $V_{0}^{*}=60$. Electrons trajectories for (b) $T=10 \mathrm{mK}$ and $F^{*}=3$, (c) $T=10 \mathrm{mK}$ and $F^{*}=150$, and (d) $T=40 \mathrm{mK}$ and $F^{*}=150$

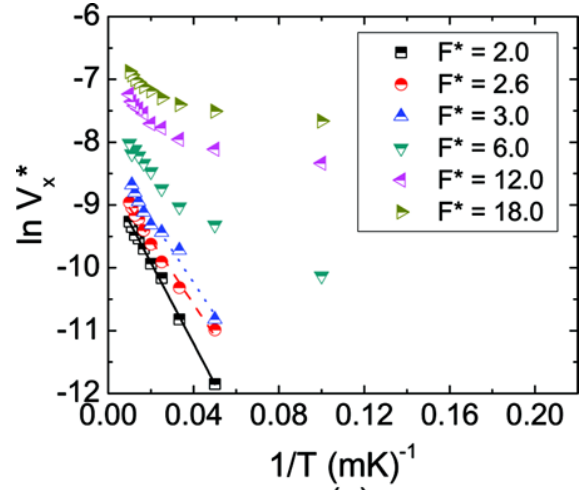

(a)

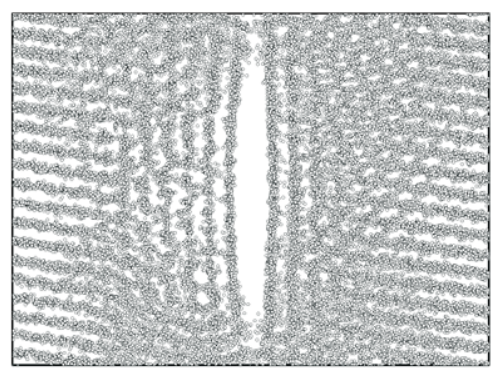

(b) $\mathrm{T}=10 \mathrm{mK} \mathrm{F}^{*}=3$

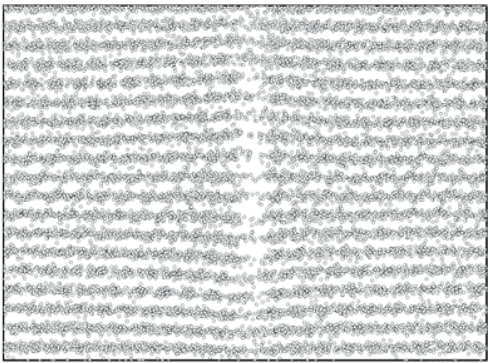

(c) $\mathrm{T}=10 \mathrm{mK} \quad \mathrm{F}^{*}=150$

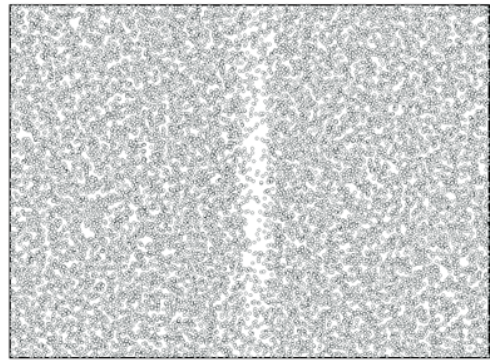

(d) $\mathrm{T}=40 \mathrm{mK} \mathrm{F}^{*}=150$ 
Fig. 4 (Color online) Scaling behavior $v_{x}^{*}=f^{* \xi}$ of current with reduced voltage $f^{*}=\left(F^{*}-F_{c}^{*}\right) / F_{c}^{*}$ for six different temperatures at $V_{0}^{*}=60$. The inset shows the temperature dependence of the exponent $\xi$

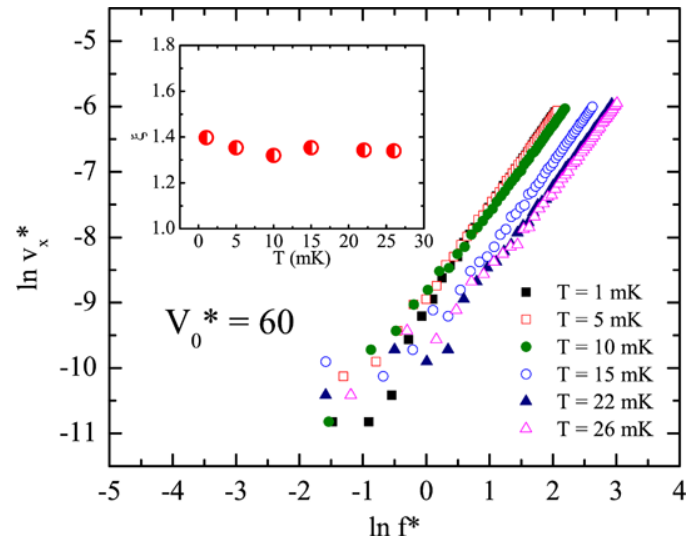

Fig. 5 (Color online) Scaling behavior $v_{x}^{*}=f^{* \xi}$ of current with reduced voltage $f^{*}=\left(F^{*}-F_{c}^{*}\right) / F_{c}^{*}$ for several values of the potential barrier height at $T=10 \mathrm{mK}$. The inset shows the barrier height dependence of the exponent $\xi$

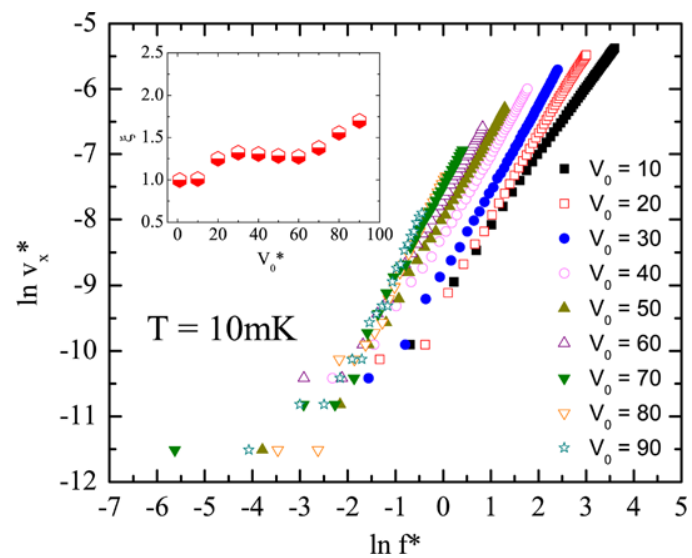

agrees with the mean-field prediction [17] and experimental results for conduction threshold in quantum dot arrays [40]. On the other hand, when the barrier height varies, at a fixed temperature of $T=10 \mathrm{mK}$ the critical exponent $\xi$ increases from $\sim 1.0$ up to $\sim 1.7$ (inset of Fig. 5). This step-like behavior is a clear indication of a crossover from quasielastic to plastic depinning. Such crossover was also observed in colloidal systems [18]. The quasielastic depinning ( $\xi$ close to unity) is an intermediate regime between elastic $(\xi=2 / 3)$ and plastic $(\xi=3 / 2)$ [41]. High values of $V_{0}^{*}$ lead to a complex arrangement of channels, in which the number of nearest neighbors of each particle changes. This is known as plastic flow regime. For high values of potential barrier, the exponent is in excellent agreement with experimental [37] and analytical [11] results. This is strong evidence that the barrier is one reason for the spread values of $\xi$ observed in experiments.

\section{Conclusions}

On this paper we have analyzed the collective transport of a two-dimensional system of electrons on a helium film adsorbed on a substrate subject to a parallel external 
force. We have examined the influence of temperature on current vs applied drive curves and found that the scaling exponent $\xi$ is weakly dependent on temperature at lower fixed values of $V_{0}^{*}$. However, there is a strong dependence of the scaling exponent on the pinning potential, with values ranging from $\sim 1.0$ to $\sim 1$.7. These results are in agreement with the dynamical critical phenomena and show that the disorder strength can have a fundamental role in spreading the values of critical exponents in similar systems.

Acknowledgements We thank the financial support provided by the Brazilian agencies: CAPES, CNPq, FAPESP and FUNAPE-UFG.

\section{References}

1. D.C. Tsui, H.L. Storner, A.C. Gossard, Phys. Rev. Lett. 48, 1559 (1982)

2. E. Abrahams, S.V. Kravchenko, M.P. Sarachik, Rev. Mod. Phys. 73, 251 (2001)

3. S.V. Kravchenko, M.P. Sarachik, Rep. Prog. Phys. 67, 1 (2004)

4. S.V. Kravchenko, G.V. Kravchenko, J.E. Furneaux, V.M. Pudalov, M. D’Iorio, Phys. Rev. B 50, 8039 (1994)

5. A. Brass, H.J. Jensen, A.J. Berlinsky, Phys. Rev. B 39, 102 (1989)

6. R. Chitra, T. Giamarchi, P. Le Doussal, Phys. Rev. Lett. 80, 3827 (1998)

7. P.L. Doussal, T. Giamarchi, Phys. Rev. B 57, 11356 (1998)

8. M.-C. Cha, H.A. Fertig, Phys. Rev. B 50, 14368 (1994)

9. G. Blatter, M.V. Feigel'man, V.B. Geshkenbein, A.I. Larkin, V.M. Vinokur, Rev. Mod. Phys. 66, 1125 (1994)

10. G. Grüner, Rev. Mod. Phys. 60, 1129 (1988)

11. A.A. Middleton, N.S. Wingreen, Phys. Rev. Lett. 71, 3198 (1993)

12. A.N. Aleshin, H.J. Lee, S.H. Jhang, H.S. Kim, K. Akagi, Y.W. Park, Phys. Rev. B 72, 153202 (2005)

13. R. Parthasarathy, X.M.L.K. Elteto, T.F. Rosenbaum, H.M. Jaeger, Phys. Rev. Lett. 92, 076801 (2004)

14. M.G. Ancona, W. Kruppa, R.W. Rendell, A.W. Snow, D. Park, J.B. Boos, Phys. Rev. B 64, 033408 (2001)

15. A. Kristensen, K. Djerfi, P. Fozooni, M.J. Lea, P.J. Richardson, A. Santrich-Badal, A. Blackburn, R.W. van der Heijden, Phys. Rev. Lett. 77, 1350 (1996)

16. K. Shirahama, Y.P. Monarkha, K. Kono, Phys. Rev. Lett. 93, 176805 (2004)

17. D.S. Fisher, Phys. Rev. B 31, 1396 (1985)

18. C. Reichhardt, C.J. Olson, Phys. Rev. Lett. 89, 078301 (2002)

19. J. Chen, Y. Cao, Z. Jiao, Phys. Rev. E 69, 041403 (2004)

20. Ç. Kurdak, A.J. Rimberg, T.R. Ho, J. Clarke, Phys. Rev. B 57, R6842 (1998)

21. R. Parthasarathy, X.M. Lin, H.M. Jaeger, Phys. Rev. Lett. 87, 186807 (2001)

22. D.S. Fisher, Phys. Rep. 301, 113 (1998)

23. O. Narayan, D.S. Fisher, Phys. Rev. B 46, 11520 (1992)

24. M.C. Marchetti, Pramana J. Phys. 64, 1097 (2005)

25. C. Reichhardt, C.J. Olson Reichhardt, Phys. Rev. B 68, 165305 (2005)

26. C. Reichhardt, C.J. Olson, I. Martin, A.R. Bishop, Europhys. Lett. 61, 221 (2003)

27. C. Reichhardt, C.J. Olson, N. Gronbech-Jensen, F. Nori, Phys. Rev. Lett. 86, 4354 (2001)

28. F. Nori, Science 271, 1373 (1996)

29. Y.P. Monarkha, K. Kono, Two-Dimensional Coulomb Liquids and Solids (Springer, Berlin, 2004)

30. V. Syvokon, Y. Monarkha, K. Nasyedkin, S. Sokolov, J. Low Temp. Phys. 148, 169 (2007)

31. L. Cândido, J.P. Rino, N. Studart, Physica B 54, 7046 (1996)

32. P. Glasson, V. Dotsenko, P. Fozooni, M.J. Lea, W. Bailey, G. Papageorgiu, S.E. Andresen, A. Kristensen, Phys. Rev. Lett. 87, 176802 (2001)

33. Y.Z. Kovdrya, V.A. Nikolaenko, A.V. Smorodin, S.S. Sokolov, J. Low Temp. Phys. 150, 242 (2008)

34. H. Ikegami, H. Akimoto, K. Kono, Phys. Rev. Lett. 102, 046807 (2009)

35. P. Glasson, G. Papageorgiu, K. Harrabi, D.G. Rees, V. Antonov, E. Collin, P. Fozooni, P.G. Frayne, Y. Mukharsky, M.J. Lea, J. Phys. Chem. Sol. 66, 1539 (2005) 
36. C.J. daSilva, J.P. Rino, L. Cândido, Phys. Rev. B 77, 193413 (2008)

37. A.N. Aleshin, S.W. Chu, V.I. Kozub, S.W. Lee, J.Y. Lee, S.H. Lee, D.W. Kim, Y.W. Park, Curr. Appl. Phys. 5, 85 (2005)

38. C. Reichhardt, C.J.O. Reichhardt, Phys. Rev. Lett. 93, 176405 (2004)

39. S. Bogdanovich, D. Popovic, Phys. Rev. Lett. 88, 236401 (2002)

40. C.I. Duruoz, R.M. Clarke, C.M. Marcus, J.J.S. Harris, Phys. Rev. Lett. 74, 3237 (1995)

41. G. Piacente, F.M. Peeters, Phys. Rev. B 72, 205208 (2005)

42. F.M. Peeters, P.M. Platzman, Phys. Rev. Lett. 50, 2021 (1983)

43. C.J. daSilva, Doctoral Thesis, Sistemas eletrônicos em duas dimensões: desordem e resposta dinâmica Universidade Federal de São Carlos, São Paulo, Brazil (2009) 